Limit on the pion distribution amplitude

This content has been downloaded from IOPscience. Please scroll down to see the full text.

2015 J. Phys. G: Nucl. Part. Phys. 42015003

(http://iopscience.iop.org/0954-3899/42/1/015003)

View the table of contents for this issue, or go to the journal homepage for more

Download details:

IP Address: 186.217.236.152

This content was downloaded on 14/10/2015 at 20:46

Please note that terms and conditions apply. 


\title{
Limit on the pion distribution amplitude
}

\author{
E G S Luna ${ }^{1}$ and A A Natale N $^{2,3}$ \\ ${ }^{1}$ Instituto de Física, Universidade Federal do Rio Grande do Sul, Caixa Postal 15051, \\ 91501-970, Porto Alegre, RS, Brazil \\ ${ }^{2}$ Centro de Ciências Naturais e Humanas, Universidade Federal do ABC, 09210-170, \\ Santo André-SP, Brazil \\ ${ }^{3}$ Instituto de Física Teórica, UNESP, Rua Dr. Bento T. Ferraz, 271, Bloco II, \\ 01140-070, São Paulo—SP, Brazil \\ E-mail: luna@if.ufrgs.br
}

Received 1 September 2014, revised 20 October 2014

Accepted for publication 28 October 2014

Published 25 November 2014

\begin{abstract}
The pion distribution amplitude (DA) can be related to the fundamental QCD Green's functions as a function of the quark self-energy and the quark-pion vertex, which in turn are associated with the pion wave function through the Bethe-Salpeter equation. Considering the extreme hard asymptotic behavior in momentum space allowed for a pseudoscalar wave function, which is limited by its normalization condition, we compute the pion DA and its second moment. From the resulting amplitude, representing the field theoretical upper limit on the DA behavior, we calculate the photon-pion transition form factor $F_{\pi \gamma \gamma^{*}}\left(Q^{2}\right)$. The resulting upper limit on the pion transition form factor is compared with existing data published by CLEO, BaBar and Belle Collaborations.
\end{abstract}

Keywords: non-perturbative QCD, pion distribution amplitude, hadron physics

(Some figures may appear in colour only in the online journal)

A few years ago new data were published [1,2] for the $\gamma^{*} \gamma \rightarrow \pi^{0}$ process, where one of the photons is far off mass shell (large $\left.Q^{2}\right)$ and the other one is near mass shell $\left(Q^{2} \approx 0\right)$. These measurements of the photon-pion transition form factor $F_{\pi \gamma \gamma^{*}}\left(Q^{2}\right)$, taken in the single-tagged two-photon $e^{+} e^{-} \rightarrow e^{+} e^{-} \pi^{0}$ reaction, were performed in a wide range of momentum transfer squared (4-40) $\mathrm{GeV}^{2}$ ). At sufficiently high $Q^{2}$ it is expected that the standard factorization approach can be applied [3-5] (for a review, see [6]). The amplitude for this process at high virtuality has the form 


$$
F_{\pi \gamma \gamma^{*}}\left(Q^{2}\right)=\frac{2 f_{\pi}}{3} \int_{0}^{1} \mathrm{~d} x \varphi_{\pi}(x) T_{\gamma \pi}^{H}\left(x, Q^{2}\right) .
$$

This equation is obtained assuming factorization of the pion distribution amplitude (DA) $\varphi_{\pi}(x)$ and the hard scattering amplitude $T_{\gamma \pi}^{H}\left(x, Q^{2}\right)$ given by $[7,8]$

$$
T_{\gamma \pi}^{H}\left(x, Q^{2}\right)=\frac{1}{(1-x) Q^{2}}\left[1+\mathcal{O}\left(\alpha_{s}\right)\right] .
$$

However the BaBar Collaboration data [1] seems to be in contradiction with this approach since, in accordance with perturbative QCD (pQCD), $Q^{2} F_{\pi r \gamma^{*}}\left(Q^{2} \rightarrow \infty\right)$ should be limited to the value $2 f_{\pi} \approx 0.185 \mathrm{GeV}$ [8], hereafter called the BL limit. At the same time, the Belle Collaboration data [2] presented in the same range of transferred momenta show that the pion transition form factor may not increase as fast as shown by the BaBar results.

As a consequence of these experiments there were many theoretical papers speculating why the data should (or not) obey the BL limit [9-19]. The first attempt to explain the BaBar result can be found in [20]. Among these there were proposals claiming that the pion distribution amplitude should be modified [9-11, 16], leading to a broader or flatter distribution in the place of the asymptotic form $\varphi_{\pi}^{\text {as }}(x)=6 x(1-x)$ [21]. A flat DA would be consistent with the BaBar data, although a field theoretical support for such possibility is still missing. Some papers claim that other transition form factors of heavier mesons are compatible among themselves and with the saturation required by factorization theorems obtained from pQCD $[13,22]$. However, for heavier mesons than the pion the DA may be more peaked away from the end points [12]. A common statement in all papers is the need for more data to settle this problem.

Meanwhile, the pion transition form factor is the most sensitive physical quantity to observe a non-perturbative contribution to the DA. Other quantities, for instance, like the pion form factor, may already contain a hard scattering amplitude at leading order with a soft behavior, due to the effect of extra coupling constants or gluon propagators [23]. This means that they do not lead to such a simple integral over a DA as the one shown by equation (1). As claimed in [24], we may assume that at present there is no definite conclusion on which is the asymptotic form of the pion DA, and it is possible that in the future a combined analysis of data of the processes involving pions will shed light on the pion distribution amplitude [25]. Notwithstanding, considering the possibility that a flatter pion DA seems to be favored by the BaBar data [9-11, 16], we can establish a field theoretical limit on how flat this DA can be, and, consequently, compare this limit to the experimental data. In order to do this we will study the DA dependence on the non-perturbative dynamics of the theory, and ultimately on the asymptotic behavior of the pion-quark vertex and the quark self-energy.

Like in the Nambu-Jona-Lasinio four-fermion approach, in QCD or any asymptotically free non-Abelian gauge theory, the fermion masses are dynamically generated along with bound state Goldstone bosons (the pions). The dynamical quark mass $\left(\Sigma\left(p^{2}\right)\right.$ ), giving by the Schwinger-Dyson equation is exactly identical to the pseudoscalar Bethe-Salpeter equation (BSE) at zero momentum transfer $\left(\left.\Phi_{\mathrm{BS}}^{P}(p, q)\right|_{q \rightarrow 0}\right)$, as demonstrated by Delbourgo and Scadron [26]

$$
\left.\Sigma\left(p^{2}\right) \approx \Phi_{\mathrm{BS}}^{P}(p, q)\right|_{q \rightarrow 0},
$$

which is a consequence of the fact that they are related through the Ward-Takahashi identity. 
The homogeneous BSE can be, in general, written as

$$
\Phi(k, P)=-\mathrm{i} \int_{q}^{\infty} \frac{\mathrm{d}^{4} q}{(2 \pi)^{4}} K(k ; q, P) S\left(q_{+}\right) \Phi(q ; P) S\left(q_{-}\right),
$$

where the amplitude depends on the quarks total $(P)$ and relative $(q)$ momenta, with $q_{+}=q+\eta P, q_{-}=q-(1-\eta) P$, and $0 \leqslant \eta \leqslant 1$, where $\eta$ is the momentum fraction parameter. In equation (4) $K$ is the fully amputated quark-antiquark scattering kernel, $S\left(q_{i}\right)$ are the dressed quark propagators, and the homogeneous BSE is valid on-shell, i.e. $P^{2}=0$ in the pion case. Note that we suppressed all indices (color, etc...) in equation (4).

The BSE, equation (4), is an integral equation that can be transformed into a second order differential equation. The two solutions of the differential equation can be found, for example, in $[27,28]$ and are characterized by one soft asymptotic solution

$$
\Phi_{\pi}^{R}\left(p^{2}\right) \sim \Sigma^{R}\left(p^{2} \gg \mu^{2}\right) \sim \frac{\mu^{3}}{p^{2}},
$$

and by the extreme hard high energy asymptotic behavior of a bound state wave function

$$
\Phi_{\pi}^{I}\left(p^{2}\right) \sim \Sigma^{I}\left(p^{2} \gg \mu^{2}\right) \sim \mu\left[1+b g^{2}\left(\mu^{2}\right) \ln \left(p^{2} / \mu^{2}\right)\right]^{-\gamma}
$$

where $b=\left(11 N_{c}-2 n_{f}\right) / 48 \pi^{2}, c=4 / 3$ is the Casimir eigenvalue for quarks in the fundamental representation $\left(N_{c}=3\right.$ is the number of colors, and $n_{f}$ is the number of quark flavors), and $\gamma=3 c / 16 \pi^{2} b$. The asymptotic expression shown in equation (6) was determined in the appendix of [29] and it satisfies the Callan-Symanzik equation. This last solution is constrained by the BSE normalization condition [30], which implies $\gamma>1 / 2$, or $n_{f}>5$ $[27,31]$, otherwise it is not consistent with a possible bound state solution in a $S U$ (3) nonAbelian gauge theory. We will take $n_{f}=6$ as will be explained later. This solution is one alternative to the soft one $\left(\Sigma\left(p^{2}\right) \sim 1 / p^{2}\right)$ [32] which leads to the standard DA $\varphi_{\pi}^{\text {as }}(x)$. Nowadays it is known that we may have solutions with a momentum behavior varying between equation (5) and equation (6) depending on the theory dynamics [31,33]. Note that the BSE can be transformed into a second order differential equation. This equation has two possible solutions, one that asymptotically behaves as $1 / p^{2}$ as in equation (5) and the other one as $\left[\ln \left(p^{2}\right)\right]^{-\gamma}$ (equation (6)). However this result comes out from the homogeneous BSE. The non-homogeneous BSE also includes a normalization condition, as discussed in $[27,30,31]$, that is obeyed by equation (5) but when applied to equation (6) implies $N_{f}>5$. This constraint appears because the wave function is very 'hard', i.e. decreases very slowly with the momentum and cannot be normalized (square integrable) if $\gamma<1 / 2$. This condition on $\gamma$ gives the bound $N_{f}>5$. This limit on $\gamma$ was obtained by Mandelstam in [30], in QCD for the first time in [27] and recently, in a different context, in [31]. If $N_{f}<6$ only the solution of equation (5) exists, because it would be the only one obeying the BSE normalization condition. This also means that if $N_{f} \geqslant 6$ QCD may have a chiral broken phase whose selfenergy is given by equation (6). Nowadays it is known that the chiral phase diagram for a non-Abelian theory may change considerably as we change the number of flavors. For instance, if the theory contains contributions of higher order operators it may have its quark self-energy or bound state solution varying between equations (5) and (6)as discussed in [33]. We are just saying that if $N_{f}>5$ equation (6) is a possible bound state solution, and the hardest one that we may have.

It has been argued that equation (6) may be a realistic wave function in a scenario where the chiral symmetry breaking is associated to confinement and the gluons have a dynamically 
generated mass $[31,34,35]$. This solution also appears when using an improved renormalization group approach in QCD, associated to a finite quark condensate [36], and it minimizes the vacuum energy as long as $n_{f}>5$ [37]. Moreover, this specific solution is the only one consistent with Regge-pole like solutions [28]. The important fact is that this is the hardest (in momentum space) asymptotic behavior allowed for a bound state solution in a non-Abelian gauge theory, and it is exactly for this reason that the constraint on $\gamma$ arises from the BSE normalization condition. No matter if this solution is realized in nature or not, it will lead to the flattest pion DA, any other flatter distribution than this one cannot be a realistic BSE wave function, and would not be consistent with a composite pion. A totally flat DA can only be related to a fundamental pion. A realistic DA, in principle, should be related to a solution of the BSE and should obey a normalization condition peculiar to a well behaved wave function.

The infrared behavior of the gap equation (or BSE) is approximately constant at small momenta, $\Sigma\left(p^{2} \rightarrow 0\right) \sim \mu$, where $\mu$, of order of a few hundred $\mathrm{MeV}$, is the characteristic scale of dynamical quark mass generation. In order to compute the pion DA we will perform an integral over the wave function in the full range of momenta (i.e. up to $p^{2} \rightarrow \infty$, this is why we will consider $n_{f}=6$ ). To obtain the extreme field theoretical limit on the pion DA, we shall also work with a simple interpolating expression that roughly reflects the full behavior of the 'hardest' quark self-energy (or BSE solution) discussed in the previous paragraphs, namely $[31,35]$

$$
\Sigma\left(p^{2}\right)=\mu\left[1+b g^{2}\left(\mu^{2}\right) \ln \left(\frac{p^{2}+\mu^{2}}{\mu^{2}}\right)\right]^{-\gamma} .
$$

Note that the $\mu$ factor introduced into the logarithm denominator leads to the right infrared (IR) behavior $\left(\Sigma\left(p^{2} \rightarrow 0\right)=\mu\right)$. Furthermore, this is just one possible ansatz for the full behavior of the self-energy and other possible interpolations between the IR and ultraviolet (UV) behaviors are possible, but as long as $\Sigma\left(p^{2}\right)$ shows the logarithmic UV behavior our final result will not change. The coupling constant $g^{2}$ is calculated at the chiral symmetry breaking scale $\mu$, and given by

$$
g^{2}\left(k^{2}\right)=\frac{1}{b \ln \left[\left(k^{2}+4 m_{g}^{2}\right) / \Lambda_{\mathrm{QCD}}^{2}\right]},
$$

which is an infrared finite coupling determined in QCD where gluons have an effective dynamical mass $m_{g}$ [38] and is consistent with the models of $[31,34,35] . \Lambda_{\mathrm{QCD}}$ is the QCD characteristic scale.

Within this approach, the pion distribution amplitude at leading twist, as a function of the quark self-energy and the pion-quark vertex, is given by [39]

$$
\begin{aligned}
\varphi_{\pi}(x)= & \frac{N_{c}}{4 \pi^{2} f_{\pi}^{2}} \int_{-\infty}^{\infty} \frac{\mathrm{d} \lambda}{2 \pi} \int_{0}^{\infty} \mathrm{d} u \frac{F(u+\mathrm{i} \lambda \bar{x}, u-\mathrm{i} \lambda x)}{D(u-\mathrm{i} \lambda x) D(u+\mathrm{i} \lambda \bar{x})} \\
& \times[x \Sigma(u+\mathrm{i} \lambda \bar{x})+\bar{x} \Sigma(u-\mathrm{i} \lambda x)],
\end{aligned}
$$

where the $u$-variable plays the role of the quark transverse momentum squared, $\lambda x$ and $-\lambda \bar{x}$ are the longitudinal projections of the quark momentum on the light cone directions $(\bar{x}=(1-x)), \Sigma(u)$ is the dynamical quark mass given by $(7)$,

$$
D(u) \equiv u+\Sigma^{2}(u),
$$


and the function $F$ is the momentum dependent part of the quark-pion vertex, which can be approximated by $F\left(p^{2}, p^{\prime 2}\right)=\sqrt{\Sigma\left(p^{2}\right) \Sigma\left(p^{\prime 2}\right)}$, where $p$ and $p^{\prime}$ are the quark and anti-quark momenta. The pion DA at leading twist is normalized as

$$
\int_{0}^{1} \mathrm{~d} x \varphi_{\pi}(x)=1
$$

It is also useful to write down the expression for the pion DA in the form found in [11] (the so called Schwinger representation)

$$
\begin{aligned}
\varphi_{\pi}(x)= & \frac{N_{c}}{4 \pi^{2} f_{\pi}^{2}} \int_{0}^{\infty} \frac{\mathrm{d} L}{L} \mathrm{e}^{x \bar{x} L p^{2}}\left[x \sigma_{m}(x L) \sigma(\bar{x} L)\right. \\
& +(x \leftrightarrow \bar{x})],
\end{aligned}
$$

where $\sigma(\alpha)$ and $\sigma_{m}(\alpha)$ are the Laplace transformations of

$$
\frac{\Sigma^{1 / 2}(u)}{D(u)} \text { and } \frac{\Sigma^{3 / 2}(u)}{D(u)},
$$

correspondingly. For example,

$$
\frac{\Sigma^{1 / 2}(u)}{D(u)}=\int_{0}^{\infty} \mathrm{d} \alpha \mathrm{e}^{-\alpha u} \sigma(\alpha)
$$

For the model calculations we take the following parameters: $\mu=100 \mathrm{MeV}$, $\Lambda_{\mathrm{QCD}}=300 \mathrm{MeV}$ and $m_{g}=321.18 \mathrm{MeV}$ [40]. To describe figure 1 we used $\Lambda_{\mathrm{QCD}}=300$ $\mathrm{MeV}$ and for $n_{f}=6$ a more appropriate value would be $\Lambda_{\mathrm{QCD}} \approx 200 \mathrm{MeV}$ [41]. However it should be noticed that the result is more dependent on the ratio $m_{g} / \Lambda_{\mathrm{QCD}}$ than on the proper $\Lambda_{\mathrm{QCD}}$ value. We also emphasize that the largest origin of uncertainty in our result is the assumption of equation (7) for the self-energy in the full range of momenta. In the intermediate and infrared region of momenta equation (7) may give a poor description of the selfenergy, although the good point is that the flat DA behavior is totally credited to the hard asymptotic self-energy behavior. Within the model considered we take the expression for the pion decay constant in the so-called Pagels-Stokar form [42]

$$
f_{\pi}^{2}=\frac{N_{c}}{4 \pi^{2}} \int_{0}^{\infty} \mathrm{d} u \frac{u \Sigma(u)}{D^{2}(u)}\left(\Sigma(u)-\frac{1}{2} u \Sigma^{\prime}(u)\right),
$$

where $\Sigma^{\prime}(u)=\mathrm{d} \Sigma(u) / \mathrm{d} u$. With the given set of parameters we obtain $f_{\pi}=92.4 \mathrm{MeV}$. Actually, it is possible to obtain this $f_{\pi}$ value with different values for $\mu$ and $m_{g}$, just changing the formula that interpolates between the IR and UV regimes, although these values should stay around a few hundreds of $\mathrm{MeV}$.

The pion DA obtained with above parameters and equation (7) and (9) is shown in figure 1; for comparison we also draw the asymptotic wave function $\varphi_{\pi}^{\text {as }}(x)$. The DA turns out to be quite flat, and we have not observed any significant variation as we change $m_{g}$ and $\mu$ as long as we do not modify the $f_{\pi}$ value in equation (9) and maintain the logarithmic UV behavior, which is at the origin of the flat DA behavior. The DA flatness is totally dependent on the logarithmic behavior of the self-energy.

The asymptotic behavior as $x \rightarrow 0$ for the model considered here is given by

$$
\varphi_{\pi}(x \rightarrow 0) \sim\left(\ln \frac{1}{x}\right)^{-\gamma / 2},
$$




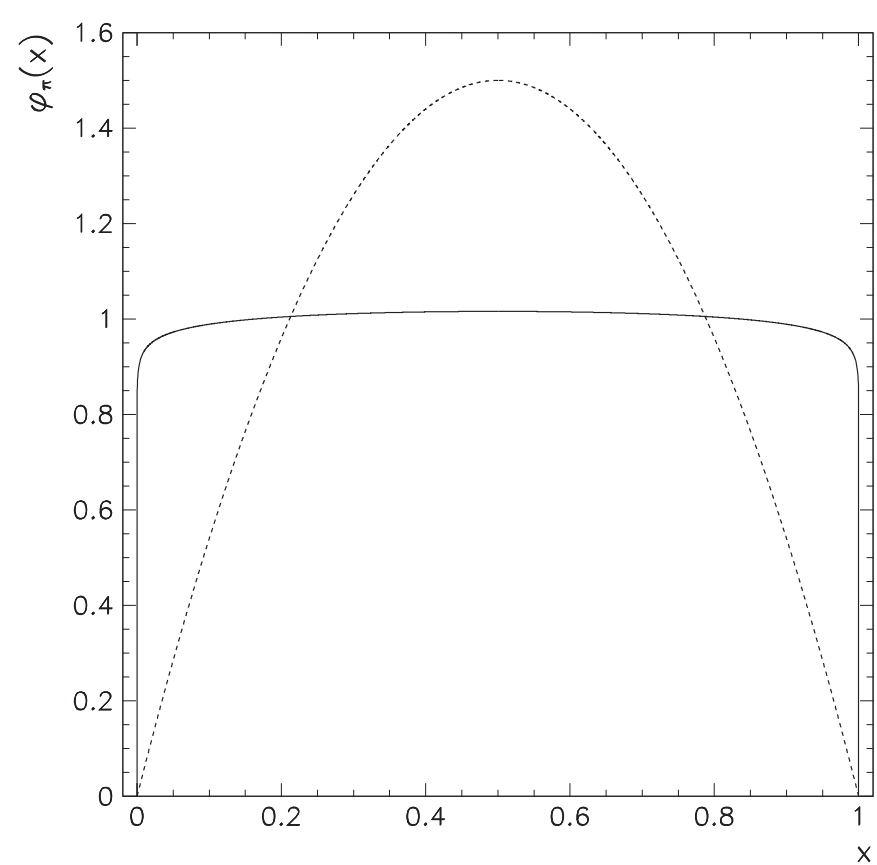

Figure 1. Pion DA computed with equations (9) and (7), and using the parameters $m_{g}=322 \mathrm{MeV}$ and $\mu=100 \mathrm{MeV}$ (solid curve). The perturbative-QCD asymptotic pion DA, $\varphi_{\pi}^{\text {as }}(x)=6 x(1-x)$, is shown by the dashed curve for comparison.

where $\varphi_{\pi}(x=0)=\varphi_{\pi}(x=1)=0$. This behavior is actually the expected one for a pion DA with a vertex function $F\left(p^{2}, p^{\prime 2}\right)$ similar to ours, where $F\left(p^{2}, p^{\prime 2}\right)$ goes to zero in the limit $p^{\prime} \rightarrow \infty$ [11]. In the appendix we determine the asymptotic behavior shown in equation (15). form

Our pion DA numerical result can be reasonably reproduced by using the normalized

$$
\varphi_{\pi}(x)=\frac{\Gamma(2+2 \epsilon)}{\Gamma^{2}(1+\epsilon)} x^{\epsilon}(1-x)^{\epsilon},
$$

where $\epsilon \approx 0.024802$. However, it is worth noting that the calculations performed in this work have been carried out using numerical values of the pion DA obtained from equation (9).

The leading asymptotic behavior of the form factor is expressed through the pion DA (16) as [11]

$$
F_{\pi \gamma^{*} \gamma}\left(0 ; Q^{2}, 0\right) \stackrel{Q^{2} \rightarrow \infty}{=} \frac{2}{3} f_{\pi} \int_{0}^{1} \mathrm{~d} x \frac{\varphi_{\pi}(x)}{x Q^{2}} .
$$

If we were considering a totally flat DA this integral would diverge. However, as emphasized by Radyushkin [9], the finite size $R \approx 1 / M$ of the pion should provide a cut-off for the $x$ integral. Therefore the $x Q^{2}$ in the denominator of equation (17) will be changed as

$$
x Q^{2} \rightarrow x Q^{2}+M^{2}
$$

In principle the factor $M$ should be related to the dynamical quark mass. It was also proposed by Radyushkin that $M$ could be treated as an effective gluon mass. Indeed the meson radius may have a deep connection with the effective gluon mass as discussed in [43]. Therefore, 


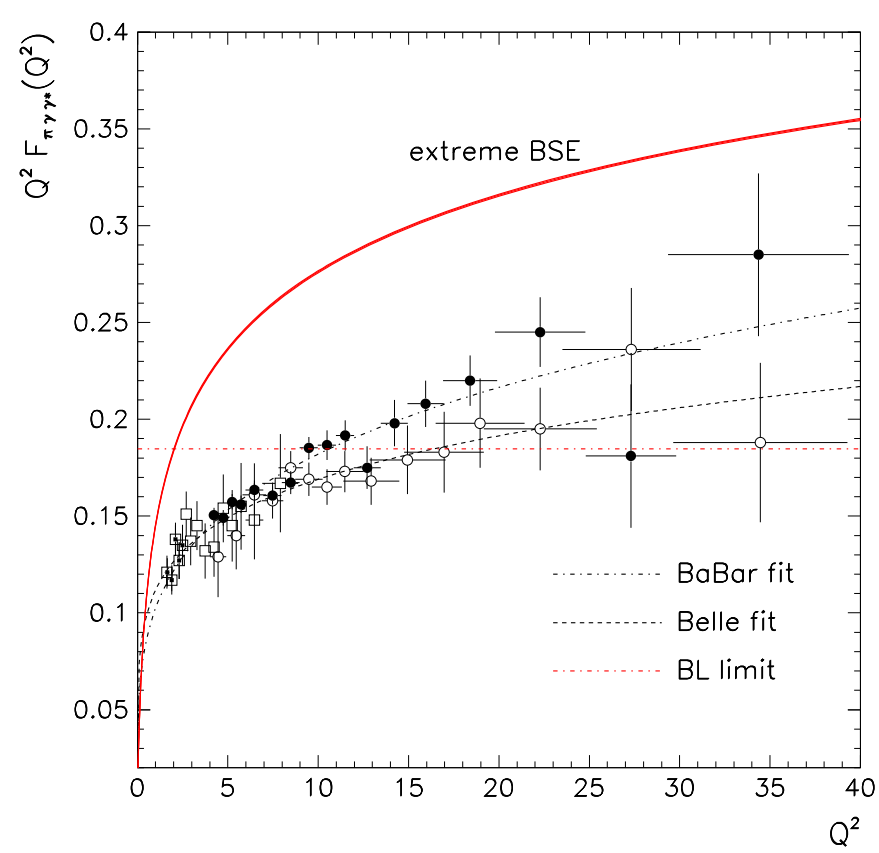

Figure 2. The photon-pion transition form factor $F_{\pi \gamma \gamma^{*}}\left(Q^{2}\right)$ computed with the extreme DA calculated in this work. The continuous red curve is the limit obtained with equation (19). The horizontal hatched area correspond to the asymptotic BL limit. The experimental data are taken from the CLEO $(\square)$, Belle (०) and BaBar (•) Collaborations.

regardless of if we have one case or another, the transition form factor will be given by

$$
F_{\pi \gamma{ }^{*} \gamma}\left(0 ; Q^{2}, 0\right)=\frac{2}{3} f_{\pi} \int_{0}^{1} \mathrm{~d} x \frac{1}{x Q^{2}+M^{2}} .
$$

$M$ being a dynamical mass should have a momentum dependence showing the decrease of the mass with the momentum. However when $x Q^{2}$ is small we can safely substitute $M\left(x Q^{2}\right)$ by $M$ in equation (19), and for large $x Q^{2}$ the momentum behavior of $M\left(x Q^{2}\right)$ is negligible. The result for the transition form factor given by equation (19) is shown in figure 2. In figure 2 we are just assuming $M=320 \mathrm{MeV}$ regardless of if this is a quark or gluon dynamical mass. We are neglecting the effects of the QCD evolution in equation (19) and, consequently, in figure 2, because, as verified by Radyushkin [9], these effects are small for such a flat DA.

The result of figure (2) for the photon-pion transition form factor can be compared to the parameterization fit of the BaBar [1] and Belle [2] Collaborations,

$$
Q^{2}\left|F_{\pi \gamma \gamma^{*}}\left(Q^{2}\right)\right|=A\left(\frac{Q^{2}}{10 \mathrm{GeV}^{2}}\right)^{\beta}
$$

where $A=0.182 \pm 0.002 \mathrm{GeV}$ and $\beta=0.25 \pm 0.02$ for $\mathrm{BaBar}$, and $A=0.169 \pm 0.006$ $\mathrm{GeV}$ and $\beta=0.18 \pm 0.05$ for Belle.

With our numerical pion DA we can also compute the second moment of the DA, which is given by 


$$
\left\langle\xi^{2}\right\rangle\left(Q^{2}\right)=\int_{0}^{1} \mathrm{~d} x \varphi_{\pi}\left(x, Q^{2}\right) \xi^{2},
$$

where $\xi=(2 x-1)$. Our result can be compared to the lattice value $\left\langle\xi^{2}\right\rangle=0.28(1)(2)$ [44], where the first error is statistical and the second is systematic. The $\left\langle\xi^{2}\right\rangle$ lattice value has been computed at the scale $Q^{2}=2.5 \mathrm{GeV}^{2}$, while our DA calculation is obtained at the scale of the chiral symmetry breaking. Since the DA evolution with the momentum can only diminish the IR value of the integral in equation (21) we can consider our result as an upper limit on the value of the second moment of the DA, i.e.

$$
\left\langle\xi^{2}\right\rangle<0.329,
$$

which is the highest value for all the parameters that we have considered.

Radyushkin [9] and Polyakov [10] have proposed a flat behavior for the pion DA in the light of BaBar data. This behavior, if it indeed happens in nature, should be a justified field theoretically. We have verified that a flat behavior for the pion DA is totally dependent on the asymptotic UV logarithmic behavior of the self-energy. When the pion DA is calculated with equation (9) using softer quark self-energies the result turns out to be more peaked at $x=0.5$. The effect of the self-energy behavior at small and intermediate momenta are erased by the normalization condition equation (11).

The high energy limit of the pion transition form factor with a flat DA must have a natural cut-off $(M)$ as proposed by Radyushkin [9] and Polyakov [10], and this one was introduced by us in equation (19). We have assumed a cut-off of the order of the dynamical masses of the theory, while in $[9,10]$ these values are quite high and difficult to associate to some physical scale. This is why our calculation can only be considered as an upper limit on the pion transition form factor. If we had assumed a larger $M$ value we would have a better agreement with the BaBar data. Of course, radiative corrections may also bring down the red curve in figure 2 implying a better adjust of the data. Hence our result provides a field theoretical limit on the pion DA and transition form factor based on the hardest asymptotic quark self-energy allowed in QCD. We also obtained a limit on the second moment of the pion DA compatible with the one of recent QCD lattice simulation.

\section{Acknowledgments}

We are grateful to A E Dorokhov for many discussions and the collaboration in the appendix calculation. This research was partially supported by the Conselho Nacional de Desenvolvimento Científico e Tecnológico (CNPq), by Coordenação de Aperfeiçoamento do Pessoal de Ensino Superior (CAPES), by grant 2013/22079-8 of Fundação de Amparo à Pesquisa do Estado de São Paulo (FAPESP), and by Fundação de Amparo à Pesquisa do Estado do Rio Grande do Sul (FAPERGS).

\section{Appendix}

Let us find the small $x$ behavior of $\varphi_{\pi}(x)$ by using the Schwinger representation (12). We also make the following approximation for the denominators of the integrand

$$
D\left(p^{2}\right)=p^{2}+\Sigma^{2}\left(p^{2}\right) \rightarrow D_{1}\left(p^{2}\right)=p^{2}+\mu^{2} .
$$


Then, first we need to find the Laplace transformation of the factor $\Sigma^{1 / 2}\left(p^{2}\right) / D_{1}\left(p^{2}\right)$

$$
\begin{aligned}
& \frac{\Sigma^{1 / 2}\left(p^{2}\right)}{D_{1}\left(p^{2}\right)}=\frac{\mu^{1 / 2}}{p^{2}+\mu^{2}} \frac{1}{\left[1+\bar{b} \ln \left(\frac{p^{2}+\mu^{2}}{\mu^{2}}\right)\right]^{\gamma / 2}} \\
& =\frac{\mu^{1 / 2}}{p^{2}+\mu^{2}} \frac{1}{\Gamma_{\gamma / 2}} \int \mathrm{d} \alpha \alpha^{\gamma / 2-1} \\
& \times \exp \left[-\alpha\left(1+\bar{b} \ln \left(\frac{p^{2}+\mu^{2}}{\mu^{2}}\right)\right)\right] \\
& =\frac{\mu^{1 / 2}}{p^{2}+\mu^{2}} \frac{1}{\Gamma_{\gamma / 2}} \int \mathrm{d} \alpha \alpha^{\gamma / 2-1} e^{-\alpha}\left(\frac{\mu^{2}}{p^{2}+\mu^{2}}\right)^{\alpha \bar{b}} \\
& =\frac{\mu^{1 / 2}}{\Gamma_{\gamma / 2}} \int \mathrm{d} \alpha \alpha^{\gamma / 2-1} \mathrm{e}^{-\alpha}\left(\mu^{2}\right)^{\alpha \bar{b}} \\
& \times \frac{1}{\Gamma(1+\alpha \bar{b})} \int \mathrm{d} \beta \mathrm{e}^{-\beta\left(p^{2}+\mu^{2}\right)} \beta^{\alpha \bar{b}} \\
& =\frac{\mu^{1 / 2}}{\bar{b}^{\gamma / 2}} \int \mathrm{d} \beta \mathrm{e}^{-\beta p^{2}} \mathrm{e}^{-\beta \mu^{2}} \\
& \times \int_{0}^{\infty} \mathrm{d} \alpha \mathrm{e}^{-\alpha / \bar{b}} \frac{\alpha^{\gamma / 2-1}\left(\mu^{2} \beta\right)^{\alpha}}{\Gamma_{\gamma / 2} \Gamma(1+\alpha)} \\
& \equiv \int \mathrm{d} \beta \mathrm{e}^{-\beta p^{2}} G_{1 / 2}(\beta),
\end{aligned}
$$

where $\bar{b} \equiv b g^{2}$ and

$$
G_{1 / 2}(\beta)=\mathrm{e}^{-\beta \mu^{2}} \frac{\mu^{1 / 2}}{\bar{b}^{\gamma / 2}} \frac{1}{\Gamma_{\gamma / 2}} \int_{0}^{\infty} \mathrm{d} \alpha \mathrm{e}^{-\alpha / \bar{b}} \frac{\alpha^{\gamma / 2-1}\left(\mu^{2} \beta\right)^{\alpha}}{\Gamma(1+\alpha)}
$$

Similarly we obtain the Laplace transform of the factor $\Sigma^{3 / 2}\left(p^{2}\right) / D_{1}\left(p^{2}\right)$ as

$$
G_{3 / 2}(\beta)=\mathrm{e}^{-\beta \mu^{2}} \frac{\mu^{3 / 2}}{\bar{b}^{3 \gamma / 2}} \frac{1}{\Gamma_{3 \gamma / 2}} \int_{0}^{\infty} \mathrm{d} \alpha \mathrm{e}^{-\alpha / \bar{b}} \frac{\alpha^{3 \gamma / 2-1}\left(\mu^{2} \beta\right)^{\alpha}}{\Gamma(1+\alpha)}
$$

Next we substitute these expressions in (12) keeping in mind that we are interested in the $x \rightarrow 0$ behavior. Then one has for the integrand 


$$
\begin{aligned}
\bar{x} G_{0, m}(x L, \bar{x} L)= & \bar{x} G_{1 / 2}(x L) G_{3 / 2}(\bar{x} L) \\
& \stackrel{x \rightarrow 0}{\approx} \mathrm{e}^{-L \mu^{2}} \frac{\mu^{2}}{\bar{b}^{2 \gamma} \Gamma_{\gamma / 2} \Gamma_{3 \gamma / 2}} \\
& \times \int_{0}^{\infty} \mathrm{d} \alpha \mathrm{e}^{-\alpha / \bar{b}} \frac{\alpha^{\gamma / 2-1}\left(\mu^{2} x L\right)^{\alpha}}{\Gamma(1+\alpha)} \\
& \times \int_{0}^{\infty} \mathrm{d} \beta \mathrm{e}^{-\beta / \bar{b}} \frac{\beta^{3 \gamma / 2-1}\left(\mu^{2} L\right)^{\beta}}{\Gamma(1+\beta)}
\end{aligned}
$$

and for the pion DA

$$
\begin{aligned}
\varphi_{\pi}(x \rightarrow 0)= & \frac{N_{c}}{4 \pi^{2} f_{\pi}^{2}} \int_{0}^{\infty} \frac{\mathrm{d} L}{L} G_{0, m}(x L, \bar{x} L) \\
= & \frac{N_{c}}{4 \pi^{2} f_{\pi}^{2}} \int_{0}^{\infty} \frac{\mathrm{d} L}{L} \mathrm{e}^{-L \mu^{2}} \frac{\mu^{2}}{\bar{b}^{2 \gamma} \Gamma_{\gamma / 2} \Gamma_{3 \gamma / 2}} \\
& \times \int_{0}^{\infty} \mathrm{d} \alpha \mathrm{e}^{-\alpha / \bar{b}} \frac{\alpha^{\gamma / 2-1}\left(\mu^{2} x L\right)^{\alpha}}{\Gamma(1+\alpha)} \\
& \times \int_{0}^{\infty} \mathrm{d} \beta \mathrm{e}^{-\beta / \hbar} \frac{\beta^{3 \gamma / 2-1}\left(\mu^{2} L\right)^{\beta}}{\Gamma(1+\beta)} \\
= & \frac{N_{c}}{4 \pi^{2} f_{\pi}^{2}} \frac{\mu^{2}}{\bar{b}^{2 \gamma} \Gamma_{\gamma / 2} \Gamma_{3 \gamma / 2}} \int_{0}^{\infty} \mathrm{d} \alpha \mathrm{e}^{-\alpha / \bar{b}} \alpha^{\gamma / 2-1} x^{\alpha} f(\alpha),
\end{aligned}
$$

where

$$
f(\alpha)=\int_{0}^{\infty} \mathrm{d} \beta \mathrm{e}^{-\beta / \bar{b}} \beta^{3 \gamma / 2-1} \frac{\Gamma(\alpha+\beta)}{\Gamma(1+\alpha) \Gamma(1+\beta)} .
$$

In the asymptotic regime $x \rightarrow 0$, we can take $f(\alpha)$ at $\alpha=0$ and obtain

$$
\varphi_{\pi}(x \rightarrow 0) \sim \frac{N_{c}}{4 \pi^{2} f_{\pi}^{2}} \frac{\mu^{2}}{\bar{b}(3 \gamma / 2-1)}\left(1+\bar{b} \ln \frac{1}{x}\right)^{-\gamma / 2} .
$$

This is the result given above in equation (15). Note, that this last result is proportional to $\Sigma^{1 / 2}$ with the argument $\left(p^{2}+\mu^{2}\right) / \mu^{2}$ of $\log$ substituted by $1 / x$. If $\gamma \leqslant 2$ then the convergence to this asymptotic behavior is rather slow.

\section{References}

[1] Aubert B et al BaBar Collaboration 2009 Phys. Rev. D 80052002 (arXiv:0905.4778)

[2] Uehara S et al Belle Collaboration 2012 Phys. Rev. D 86092007 (arXiv:1205.3249 [hep-ex])

[3] Chernyak V L and Zhitnitsky A R 1977 JETP Lett. 25510

[4] Lepage G P and Brodsky S J 1979 Phys. Lett. B 87359

[5] Efremov A V and Radyushkin A V 1980 Phys. Lett. B 94245

[6] Brodsky S J and Lepage G P 1989 Adv. Ser. Direct. High Energy Phys. 593

[7] Brodsky S J and Lepage G P 1981 Phys. Rev. D 241808

[8] Lepage G P and Brodsky S J 1980 Phys. Rev. D 222157

[9] Radyushkin A V 2009 Phys. Rev. D 80094009 (arXiv:0906.0323) 
[10] Polyakov M V 2009 JETP Lett. 90228 (arXiv:0906.0538)

[11] Dorokhov A E 2010 JETP Lett. 92707

[12] Dorokhov A E 2012 Nucl. Phys. Proc. Suppl. 225-227 141 (arXiv:1109.3754 [hep-ph])

[13] Mikhailov S V and Stefanis N G 2009 Nucl. Phys. B 821291 (arXiv:0905.4004)

[14] Brodsky S J, Cao F-G and de Teramond G F 2011 Phys. Rev. D 84033001 (arXiv:1104.3364 [hep-ph])

[15] Ruiz Arriola E and Broniowski W 2010 Phys. Rev. D 81094021 (arXiv:1004.0837 [hep-ph])

[16] Noguera S and Vento V 2010 Eur. Phys. J. A 46197 (arXiv:1001.3075 [hep-ph])

[17] Agaev S S, Braun V M, Offen N and Porkert F A 2011 Phys. Rev. D 83054020 (arXiv:1012.4671 [hep-ph])

[18] Kroll P 2011 Eur. Phys. J. C 711623 (arXiv:1012.3542 [hep-ph])

[19] Klopot Y, Oganesian A and Teryaev O 2013 Phys. Rev. D 87036013 (arXiv:1211.0874 [hep-ph])

[20] Dorokhov A E 2010 Phys. Part. Nucl. Lett. 7229 (arXiv:0905.4577 [hep-ph])

[21] Efremov A V and Radyushkin A V 1980 Theor. Math. Phys. 4297

[22] Stefanis N G, Bakulev A P, Mikhailov S V and Pimikov A V 2013 Phys. Rev. D 87094025 (arXiv:1202.1781)

[23] Aguilar A C, Mihara A and Natale A A 2002 Phys. Rev. D 65054011

[24] Wu X-G and Huang T 2010 Phys. Rev. D 82034024

[25] Huang T, Zhong T and Wu X-G 2013 Phys. Rev. D 88034013

[26] Delbourgo R and Scadron M D 1979 J. Phys. G: Nucl. Part. Phys. 51621

[27] Lane K 1974 Phys. Rev. D 102605

[28] Langacker P 1975 Phys. Rev. Lett. 341592

[29] Cornwall J M and Shellard R C 1978 Phys. Rev. 181216

[30] Mandelstam S 1955 Proc. R. Soc. A 233248

Llewellyn Smith C H 1969 Nuovo Cimento A 60348

[31] Doff A, Luna E G S and Natale A A 2013 Phys. Rev. D 88055008

[32] Politzer H D 1976 Nucl. Phys. B 117397

[33] Takeuchi T 1989 Phys. Rev. D 402697

Kondo K-I, Shuto S and Yamawaki K 1991 Mod. Phys. Lett. A 63385

[34] Doff A, Machado F A and Natale A A 2012 Annals Phys. 3271030

[35] Doff A, Machado F A and Natale A A 2012 New J. Phys. 14103043

[36] Chang L-N and Chang N-P 1984 Phys. Rev. D 29312

Chang L-N and Chang N-P 1985 Phys. Rev. Lett. 542407

Chang N-P and Li D S 1984 Phys. Rev. D 30790

[37] Montero J C, Natale A A, Pleitez V and Novaes S F 1985 Phys. Lett. B 161151

[38] Cornwall J M 1982 Phys. Rev. D 261453

[39] Dorokhov A E 2003 JETP Lett. 7763

Dorokhov A E 2003 Pisma Zh. Eksp. Teor. Fiz. 7768 (arXiv:hep-ph/0212156)

[40] Halzen F, Krein G I and Natale A A 1993 Phys. Rev. D 47295

Luna E G S 2006 Phys. Lett. B 641171

Natale A A 2009 PoS QCD-TNT 09031

Luna E G S, dos Santos A L and Natale A A 2011 Phys. Lett. B 69852

Luna E G S and Beggio P C 2014 Nucl. Phys. A 929230

[41] Bethke S 2013 Nucl. Phys. Proc. Suppl. 234229

[42] Pagels H and Stokar S 1979 Phys. Rev. D 202947

[43] Gay Ducati M B, Halzen F and Natale A A 1993 Phys. Rev. D 482324

[44] Arthur R et al 2011 Phys. Rev. D 83074505 